\title{
Is Daily Awakening Always Safe in Severely Brain Injured Patients?
}

\author{
Raimund Helbok $\cdot$ Neeraj Badjatia
}

Published online: 8 August 2009

(C) Humana Press Inc. 2009

Sedation is a central component of critical care to prevent sleep deprivation, pain, anxiety, agitation, and delirium. Daily interruption of sedation (DIS) decreases the duration of mechanical ventilation, shortens hospital stay and may, in combination with spontaneous breathing trials, improve outcome in medical intensive care patients [1,2]. Moreover, the amount of sedatives and opioids administered, which is directly associated with morbidity (nosocomial infections and thromboembolic events), can be reduced by DIS [1,2]. Therefore, sedative drugs should be titrated to a lower level when sedation is restarted, which is, in our mind, the major factor contributing to decrease ventilation and ICU days. An estimated 30-40\% of intensive care units worldwide have implemented daily interruption of sedation as a routine protocol [3-5]. Even if daily interruption of sedation could be a cornerstone for medical intensive care patients, it has not extensively been studied so far in acutely brain injured patients. So, can these results be translated to patients with subarachnoidal hemorrhage (SAH), intracerebral hemorrhage (ICH), or traumatic brain injury (TBI)? In fact, in a recent randomized control trial, the subgroup of acutely head injured patients did not show decreased ventilation or ICU

\section{R. Helbok $(\varangle) \cdot$ N. Badjatia}

Division of Critical Care Neurology, Department of Neurology,

Milstein Hospital 8 Center, Columbia University, 177 Fort

Washington Ave, New York, NY 10032, USA

e-mail: raimund.helbok@uki.at

N. Badjatia

e-mail: nb2217@columbia.edu

\section{R. Helbok}

Clinical Department of Neurology, Neurological Intensive Care Unit, Medical University Innsbruck, Anichstrasse 35, 6020 Innsbruck, Austria days when sedation was interrupted on a daily basis $(n=21)$ compared to controls $(n=17)$ [6].

The major reason for neurointensivists to prescribe sedative drugs is to decrease oxygen consumption and prevent metabolic distress in the severely injured brain. Unfortunately, continuous sedation can mask important changes that can only be elucidated by clinical exam, which remains so far the gold standard. Therefore, several NICUs in the USA have already implemented the strategy of daily or even twice daily awakening for reliable assessment. Immediate detection of early (brain edema, rebleeding) and late (e.g., delayed cerebral ischemia secondary to cerebral vasospasm) complications in the course of SAH, ICH, and TBI may improve outcome. Modern advances in neurocritical care has led to the implementation of multimodal neuromonitoring, which includes monitoring of ICP (intraparenchymal or with EVD), cerebral metabolism (microdialysis), brain tissue oxygen (PbtO2), and cerebral blood flow (CBF). This may help to early detect complications, however, so far, we are still on the way to introduce these data in the daily care of our patients. So, relying on clinical evaluation by stopping sedatives, do we know whether this is safe for NICU patients or may the risk of metabolic derangement overweigh the benefit of an appropriate exam?

The study by Skoglund and coworkers in this issue of the Journal is the first effort to prove safety of daily interruption of sedation in NICU patients. A total of 127 awakening trials with interruption of continuous propofol infusion were studied in $12 \mathrm{TBI}$ and 9 SAH patients. In the majority of patients (13/21) the authors observed an increase in ICP (to mean $22 \pm 7 \mathrm{mmHg}$ ) and CPP (to mean $79 \pm 21 \mathrm{mmHg}$ ). In 23 awakening trials (8 patients) a decrease in CPP was observed as ICP increased $(\geq 25 \mathrm{mmHg}$ ). No other adverse events were reported, and 
the authors conclude that awakening trials are safe in the majority of SAH and TBI patients, however, further trials using multimodality neuromonitoring may be needed.

These conclusions, however, lead to other questions and also emphasize study limitations. First, the authors argue that daily awakening seems safe in their experience, although a certain number of patients developed ICP crisis during the trial, requiring immediate administration of propofol and morphine. Raised intracranial pressure is associated with brain metabolic distress and poor outcome in both TBI and SAH patients, however, the duration and etiology may be of importance. In 8 patients (38\%) CPP considerably decreased to a level below $50 \mathrm{mmHg}$ for mean $10.2 \pm 17$ min secondary to ICP elevation. Current guidelines state to avoid CPP $<50$ in TBI (Level III) [7] and even recommend higher CPP in patients with symptomatic cerebral vasospasm [8]. Adequate brain perfusion is essential to establish continuous energy supply. A decrease in cerebral oxygenation and brain glucose may be harmful for the brain at risk and cause metabolic distress and ischemia. Whether a trial of daily interruption of sedation can cause such a cascade still has to be proven. Moreover, cerebral autoregulation may play a key role: The 14\% increase in MAP reported by Skoglund and coworkers may result in decreased ICP with the price of cerebral vasoconstriction when autoregulation is preserved. A higher MAP can also result in higher CBF when autoregulation is impaired. Patients with severe vasospasm may benefit from improved microcirculation, however, others may deteriorate secondary to an increase in ICP.

Another limitation is the exclusion of non-stable patients from DIS in the current trial: are not these the patients we are most interested in, to detect clinical deterioration? The benefits of daily interruption of sedation may not come without a price in all neurological patients. We may have to pay the price of metabolic distress in these severely brain injured patients.

One of the major rationale why certain neurointensivist believe in the benefit of daily awakening trials is the additional information gained from a reliable clinical assessment. However, Skloglund and coworkers neither reported the change in clinical exam when sedation was stopped nor discussed the value they gained in their patients. The central question is: how many patients with clinical deterioration were identified leading to change in patients' management? If there is no benefit, a sedation algorithm guided by commonly used sedation scales alone may be sufficient in NICU patients? However, the problem with this approach is, that we still lack of validated sedation scales to guide the neurointensivist in the management of acutely brain injured patients.

The authors monitored patients for only a few adverse events, including ICP, CPP, and oxygen saturation. Other important adverse events, however, were not examined. For instance, whether heart rate increased and end tidal $\mathrm{CO} 2$ levels decreased after sedation was discontinued, both of which may result in an increase in energy expenditure leading to a higher systemic and cerebral metabolic rate and a potential risk for tissue hypoxia. Hyperventilation may decrease cerebral blood flow (CBF) and limit energy supply to the brain (oxygen and glucose delivery). Aerobic metabolism may then shift to anaerobic as reflected by an increase in lactate-pyruvate ratio (LPR). Moreover, cerebral metabolic rate of oxygen (CMRO2) assessed by measurement of jugular bulb venous oxygen tension should be monitored during DIS. Based on these neuromonitoring measures, we could then validate commonly used sedation scales for acutely brain injured patients and use them as simple bedside tools to guide neurointensivists.

We can thank Skloglund and coworkers for a wake-up call for practitioners in neurological intensive care units to examine practices of interruption of sedation more critically. We need better methods of ensuring that DIS is safe in all severely brain injured patients. Individualized assessment based on neuromonitoring parameters may help us in diminishing these gaps in our knowledge.

\section{References}

1. Girard TD, Kress JP, Fuchs BD, Thomason JW, Schweickert WD, Pun BT, et al. Efficacy and safety of a paired sedation and ventilator weaning protocol for mechanically ventilated patients in intensive care (awakening and breathing controlled trial): a randomised controlled trial. Lancet. 2008;371:126-34.

2. Kress JP, Pohlman AS, O'Connor MF, Hall JB. Daily interruption of sedative infusions in critically ill patients undergoing mechanical ventilation. N Engl J Med. 2000;342:1471-7.

3. Martin J, Franck M, Sigel S, Weiss M, Spies C. Changes in sedation management in german intensive care units between 2002 and 2006: a national follow-up survey. Crit Care. 2007;11:R124.

4. Mehta S, Burry L, Fischer S, Martinez-Motta JC, Hallett D, Bowman D, et al. Canadian survey of the use of sedatives, analgesics, and neuromuscular blocking agents in critically ill patients. Crit Care Med. 2006;34:374-80.

5. Tanios MA, de Wit M, Epstein SK, Devlin JW. Perceived barriers to the use of sedation protocols and daily sedation interruption: a multidisciplinary survey. J Crit Care. 2009;24:66-73.

6. Anifantaki S, Prinianakis G, Vitsaksaki E, Katsouli V, Mari S, Symianakis A, et al. Daily interruption of sedative infusions in an adult medical-surgical intensive care unit: randomized controlled trial. J Adv Nurs. 2009;65:1054-60.

7. Bratton SL, Chestnut RM, Ghajar J, McConnell Hammond FF, Harris OA, Hartl R, et al. Guidelines for the management of severe traumatic brain injury. IX. Cerebral perfusion thresholds. J Neurotrauma. 2007;24(Suppl 1):S59-64.

8. Bederson JB, Connolly ES Jr, Batjer HH, Dacey RG, Dion JE, Diringer MN, et al. Guidelines for the management of aneurysmal subarachnoid hemorrhage: a statement for healthcare professionals from a special writing group of the stroke council, American heart association. Stroke. 2009;40:994-1025. 